\title{
TEXTSPEZIFISCHE DISTRIBUTION DER VERBALTEMPORA
}

Aus dem Buch DIE VERBALKATEGORIEN DES DEUTSCHEN von Elisabeth Leiss, weiches immer wieder zu Reflexionen und Konfrontationen anregt, führe ich als Auftakt und Leitgedanken, auf den ich noch des öfteren zurückgreifen werde, den folgenden Satz an: "Die Zeit verstehen heisst also, durch geistige Beweglichkeit das Räumliche überwinden."

Nachdem ich in meinem Aufsatz den Versuch unternehme, die Kategorie des Tempus textsortenspezifisch näher auszuleuchten (im besonderen die Tempora Futur und zukunftsbezogenes Präsens sowie Präteritum und haben-Perfekt als seine analytische syntaktische Variante), sei mir erlaubt, vorerst einige grundlegende Bemerkungen über die Eigentümlichkeiten der Verbalkategorien im allgemeinen vorzubringen, um mich danach näher der Kategorie Tempus zuzuwenden, und zwar in Form einer Konfrontation der Beschreibungs- und Erklärungsweise, wie sie im besagten Buch von Leiss (1992) vorzufinden und jener die von der slowenischen Schule der NATÜRLICHEN SYNTAX entwickelt worden ist.

Nach Leiss, ja, schon nach Jakobson, lassen sich komplexere bzw. markiertere Kategorien auf weniger komplexe / weniger markierte Kategorien zurïckführen. Aufgegriffen und weiterentwickelt wurde diese Theorie von der NTS, ${ }^{*}$ welche wie auch die Kategorisierungstheorie von Leiss, auf der die Einzelkategorien überschreitenden Zusammenhänge und die Bestimmung der zwischenkategorialen Relationen aufbaut. Es werden kategoriale Überschneidungen und Affinitäten einzelner Kategorien zueinander festgestellt. In diesem Punkt stimmen auch meine bereits des öfteren vorgeführten Thesen, die aus einer umfangreichen Datenanalyse abgeleitet worden sind, mit den Überlegungen von Leiss überein. Leiss, wie übrigens auch Jakobson, lassen sich bei der Sprachbeschreibung stark von ihrer sprachlichen Intuition leiten. In der Zusammenfassung ihrer Thesen über kategoriale Entfaltungsprozesse liest man den Satz "Die Funktion hat ein Primat gegenüber der Form". Genau dieselben Schlüsse ziehe ich selbst im Laufe meiner empirisch angelegten Untersuchungen, die verschiedene distinktive kategoriale Merkmale explizieren, vor allem was die Kategorie des Tempus, genauer der Kollokationspräferenzen der verschiedenen Möglichkeiten der Versprach-

* Natürlichkeitstheorie 
lichung des Futurs und des Präsens im Deutschen, in ersten Ansätzen aber auch der Vergangenheitstempora Präteritum und Perfekt, anbelangt.

Die beiden voneinander anfangs unbeeinflußten Theorien, speziell über die Dreigliedrigkeit des Tempussystems, wonach neben der Opposition Vergangen gegenüber Nichtvergangen auch ein grammatikalisiertes Futur vorzufinden ist, unterscheiden sich von denen vieler anderer Autoren gerade dadurch, daß sie der analytischen werden+ Infinitiv- Konstruktion den Stellenwert einer grammatikalisierten Tempuskategorie einräumen und die Behauptung, das Futur im Deutschen sei ein Modus, entschieden zurückweisen. Ich darf also vorwegnehmend feststellen, dass sich unsere theoretischen Erwägungen weitgehend decken und, was die Sprachbeschreibung anbelangt, ein sehr ähnliches Lösungsverhalten anstreben. Jene Ansätze jedoch, in denen sie sich ergänzen lassen bzw. sie als komplementär aufzufassen sind, kann man dem Vergleich beider theoretischen Postulate entnehmen. Daher fasse ich nun, bevor ich auf die diesbezügliche Argumentation von Leiss näher eingehe, meine aus umfangreichen Auszählungen grammatischer Einzelinformationen abgeleiteten Überlegungen zu kategorialen Entfaltungsprozessen und deren Koexistenz, im besonderen die des Tempus, wie folgt zusammenfassen:

1.1. Es besteht ein ständiger Konkurrenzkampf zwischen verstärkten syntaktischen Varianten einerseits und ihren weniger verstärkten bzw. schwächeren da weniger markierten Oppositionspartnern andererseits. Es muß dabei zwischen der inhaltlich angesetzten Markiertheit und der formal angesiedelten Merkmalhaftigkeit unterschieden werden. Es kommt gerade diesbezüglich oft zu vermeintlichen Störungen, die das als geltend postulierte Prinzip der Ikonizität zu widerlegen scheinen.

1.2. Die Deutungsstrategie der syntaktischen Vorgänge wird ergänzt durch die Einbeziehung der breiter angelegten ko- und kontextuellen, diskursiven, aber auch außersprachlichen Merkmale (wie etwa Kollokationsaffinitäten zu gewissen grammatischen Kategorien: z.B. Präsens mit Zukunftsbezug mit Nebensätzen, nichtdritten Personen, nonadditiven Aspektvarianten usw.; Intentionen der Diskursteilnehmer, Einsetzen des Weltwissens, das sich unter anderem im sprachökonomischen Verhalten niederschlagen kann, da vom bereits Geleisteten oder Bekannten Gebrauch gemacht wird, und desgleichen mehr...). Alle diese sprachlichen Gegebenheiten können mitunter von essentieller Bedeutung für die Herstellung von semantischen Rollen bzw. referenziellen Ebenen einzelner grammatischer Kategorien sein. Um diese Behauptung mit einem Beispiel zu illustrieren sei hier der kameleonartige Charakter des Zeitadverbums "jetzt" angeführt. Seine inhaltliche Struktur wird nämlich entweder vom betreffenden Verb definiert, wenn dieses an sich temporale Bezüge aufzuwweisen vermag, also eine grammatikalisierte, im Tempussystem fest verankerte Realisierungsmöglichkeit darstellt. Bei Kollokation mit eher neutralen Verben wird der jeweilige Zeitbezug in umgekehrter Richtung vom besagten Zeitadverb hergestellt oder redefiniert: ich beende jetzt meine Arbeit, jetzt denkt sie intensiv nach, du hast jetzt erreicht, was du wolltest usw. (= starke Abhängigkeit von der Aktionsart des jeweiligen Verbums). 
1.3. Der kategoriale Entfaltungsprozess entwickelt sich eher zu Gunsten der Grammatikalisierung von verstärkteren / markierteren, aber auch merkmalhafteren Konstruktionen, die sich, nachdem dieser Prozeß abgeschlossen worden ist, nicht mehr vorzugsweise mit komplexeren grammatischen Werten / Parametern verbinden, da sie in gewissen Zusammenhängen, aber auch nur in bestimmten Textsorten, üblich geworden sind und daher zusätzliche Markierungen redundant wären, außer, sie werden durch stilistische Vorhaben motiviert. So hat z.B. die perfektive Aktionsart die Eigenschaft, Zukunftsbezug herzustellen, also wäre das umständlichere analytische Futur in solchen Fällen vermeidbar, es sei denn, daß besonderer Nachdruck beabsichtigt wird oder etwa eine größere Distanz in Richtung Nachzeitigkeit.

1.4. Eine Konstruktion kann dann als grammatikalisiert gelten, wenn die neue Funktion bereits überzeugend überwiegt und die besagte Konstruktion relevant häufiger in markierterer grammatischer Umgebung auftritt, falls es sich um eine verstärkte Kon$\underline{\text { struktion handelt. Schwächere Konstruktionen weisen gegensätzliche Verhaltensweisen }}$ auf.

1.5. Die für gewisse Textsorten typischeren Kategorien werden von ihrer unmittelbaren grammatischen Umgebung mit weniger Aufwand aufgenommen, d.h. die Verbalisierungsvariante einer gewissen Kategorie kann für jene Textsorten als die typischere Geltung beanspruchen, wo sie weniger häufig mit markierteren grammatischen Parametern kollozieren muß, da diese für die Erzeugung der zentralen semantischen Rolle durch gewisse textuelle oder diskursive Vorleistungen neutral geworden sind. In anderen Textsorten dagegen kann man von solchen Vorleistungen nicht Gebrauch machen oder sie als produktiv einsetzen. Die Affinität gewisser grammatischer Parameter zueinander ist daher nicht arbiträr. (Konkret: Zusammenwirkung von Aktionsart und Zeitrefferenz.) Die Frequenz des Auftretens ist in typischeren Textsorten größer.

1.6. Die eruierten prozentuellen Werte erwiesen sich im Sinne der o.a. Postulate als weitgehend hypothesenfreundlich. (Die durchgeführten empirischen Untersuchungen liefern zur Zeit insgesamt annähernd 85.000 grammatische Einzelinformationen.)

Nun wieder zurück zur "Kategoriesierungstheorie" von Leiss, die ich in ihren wesentlichen Merkmalen aufgreifen möchte. Die Autorin loziert die dynamischsten sprachlichen Vorgänge in dem, wie sie treffend formuliert, "auf den ersten Blick unscharfen Raum zwischen den klarer abgezeichneten Einzelkategorien, welcher ein hohes $\mathrm{Maß}$ an Ordnung aufweist." Zwischenkategoriale Relationen sind demnach regelgeleitet. Sie behauptet weiter, daß sich alle Kategorien aus einer Grunddifferenzierung, d.h. der Basiskategorie, die vom natürlichen, egozentrischen Standpunkt des Sprachproduzenten erzeugt wird, ableiten lassen. Die Entfaltung der Differenzierung, die zum schrittweisen Aufbau der unterschiedlichen Kategorien führt, sieht auch sie in den Markiertheitsrelationen.

Laut Leiss funktioniert die Sprache als ein symbolisches und ein deiktisches bzw. indexikalisches Zeichensystem. Durch deiktische sprachliche Zeichen wird die Verbal- 
handlung / das Verbalgeschehen in bezug zur Wirklichkeit gebracht, und zwar auf Grund der Einbeziehung des sogenannten Betrachters, der als Vermittler zwischen Handlung / Geschehen und Texterzeuger "eine für die Erzeugung der semantischen Kernrolle unabdingbare Entität darstellt". Symbolische Zeichen dagegen sind kontextunabhängig.

Tempus und Modus sind stark deiktisch, daher faßt sie Leiss als komplexer auf wie etwa Aspekt als ihre Basiskategorie, die ihrerseits ein einfacheres Zeitbild darstellt. Diese Argumentation veranschaulicht sie am Beispiel des Kindspracherwerbs und des Zweitspracherwerbs. Beide bewegen sich nachweislich in Richtung Aspekt - Tempus Modus.

Diese Abfolge zu Gunsten der Komplexitätssteigerung wird als eine der wesentlichsten Eigenschaften der kategorialen Entfaltung vorausgesetzt. In diesen Punkten ist die Ähnlichkeit mit Erklärungsstrategien, wie sie von der natürlichen Syntax eingesetzt werden, kaum zu übersehen. Die wesentliche Funktion aller grammatischer Kategorien, so faßt Leiss ihre These zusammen, besteht einerseits in der Entarbitrarisierung von Sprache und andererseits in der Rekonstruktion des Ortes, von dem aus auf die Welt verwiesen wird. Nun, welche Kategorie vermag diese Funktionen besser wahrzunehmen als gerade die des Tempus? Man kann also mit grammatischen Zeichen, die die Eigenschaften des Tempus ausweisen, unabhängig von "hier" und "jetzt" auf den außersprachlichen Kontext verweisen. Der Rekonstruktion des Referenzausgangspunktes wird demnach eine entscheidende Funktion eingeräumt, weil sie durch ihre Orientierungshinweise die Einschränkung der möglichen Kontexte liefert und auf diese Weise die Einführung von Weltwissen zusätzlich zum vorgegebenen sprachlichen Wissen.

In diesem Zusammenhang spielen die Erwartungshaltungen des Adressaten eine wichtige Rolle, denn Abweichungen oder gar Verstöße gegen diese Präsuppositionen haben zunächst in der Grammatik den Status einer neuen Information, die zusätzliche Markierungen erforderlich macht.

"Einen eigenen Informationswert erzeugt auch die notwendige Linearität der materiell realisierten sprachlichen Seqünzen. Entspricht die sprachliche Sequentionalität der natürlichen, kognitiven Sequentionalität, so haben wir Formen der natürlichen, nichtarbiträren Kodierung vor uns", meint Leiss weiter. Wiederum ist die Aufgabe der Kategorien, Verstöße gegen diese natürliche Serialisierung durch zusätzliche Markierung auszugleichen.

Jede Kategorie müßte auch die Inhalte Vorher und Nachher zum Ausdruck bringen können, sowie über anaphorische und kataphorische Kapazitäten verfügen.

Leiss stellt weiter fest, indem sie die Kategorienentfaltung nachvollzieht, daß der essenzielle Inhalt der jeweils vorausgegangenen kategorialen Ebene erhalten bleibt und lediglich einer neuen Lesart unterworfen wird. Konkret heißt das, daß Kategorien auch nichtoptimal eingesetzt werden können. (Zum Beispiel das Verbinden terminativer Verben mit Kataphorik.) Solche Situationen werden durch Umkategorisierungsprozesse bzw. neue Lesarten getilgt. Dadurch entstehen vorerst Übergangskategorien, wie etwa 
Präsens in seiner Funktion als Prätempus oder das Perfekt, welches ursprünglich als perfektive Aktionsart, nicht als Tempus gelesen wurde, oder modale Lesarten beim Futur. Nach dem abgeschlossenen Grammatikalisierungsprozeß, der laut Leiss erst dann erfolgt ist, wenn alle Verben zu einer Kategorie Zugang finden (auch solche, die ursprünglich Unverträglichkeit manifestiert haben), ohne daß die grundlegende refferentielle Komponente sich wesentlich verändern würde. Diese Vorgänge bezeichnet die Autorin als kontinuierliche Reinterpretationsprozesse, die potentiell unabgeschlossen sind. Beim von mir eruierten Status des analytischen Futurs findet diese These ihre Bestätigung, was heißt, daß sich auch in diesen Punkten beide Theorien erneut begegnen und den kreativen Aspekt menschlicher Sprache, jede auf ihre Weise und mit anderer Forschungsmethode, zu definieren versuchen.

3. Nun konkret zum Tempus: Es wird als innenperspektivierende Kategorie mit phorischen Kapazitäten beschrieben. Sie ermöglicht die fiktive Lösung vom jetzt und ist äußerst aspektsensibel. Nur inhärent innenperspektivierende und imperfektive bzw. additive Aspektverben besitzen nämlich die Fähigkeit, temporale Referenzen zu erzeugen, d.h. nur sie sind prototypische Tempora. Jedoch ist eine Tempusform erst dann im System fest verankert, wenn sie auch nonadditive Aspektverben als produktiv zuläßt. Ist das nicht der Fall, handelt es sich um eine Übergangskategorie bzw. ein Prätempus, wie zum Beispiel das bereits erwähnte Präsens, das sich als Schnittpunkt von Tempus und Aspekt ausweist. (So haben z. B. perfektive Verben im Präsens Zukunftsbezug, durative/additive Verben aber Gegenwartsbezug.)

Die Kategorie Tempus erzeugt eine Trennung von Sprechzeit und Betrachtzeit, wobei die Letztere als temporal interpretierter, dem Adressaten zugewiesener Standpunkt zu verstehen ist. Der Sprecher versetzt sich fiktiv als Betrachter an den Ort der Lokalisation des Ereignisses, er wechselt also seinen Standort entweder zurück oder nach vorne. Das Präsens ist jedoch durch Gleichheit von Betrachter- und Ereignislokalisation, die als Gleichzeitigkeit gedeutet wird, zu erkennen. So ist die Tempuskategorie letztlich von räumlichen Vorstellungen abgeleitet.

Nun zu den Kodierungsmöglichkeiten der Sphäre vergangen und der Sphäre zukünftig im Einzelnen. Bei den Vergangenheitstempora liegt, auch wenn imperfektive / additive Verben in die Tempuskategorie eintreten, so expliziert Leiss ihre Thesen weiter, eine Begrenzung der Handlung vor. Genau dasselbe ist auch bei den zukunftsbezogenen Tempora der Fall. Diese Begrenzung ist aber in beiden Fällen nach einer Seite offen. Denn, "die Tempora begrenzen eine Handlung oder ein Geschehen an der Nachstelle zur Gegenwart." Auch diese Überlegung rechtfertigt die o.a. Behauptung, wonach es sich beim Präsens um ein Prätempus handelt. Es liegt nämlich auf der Hand, $\mathrm{da} ß$ besonders die morphologischen Präsensformen additiver Verben stark kontextsensitiv sind. Sie können in Abhängigkeit vom Ko- und Kontext entweder futurischen oder präsentischen Zeitbezug herstellen. Nonadditivität dagegen ist mit präsentischem Zeitbezug inkompatibel. Solche Verben erzeugen auch Modalität, sobald sie nicht mit optimaler Tempusform verwendet werden. Das analytische Futur stößt aber seinerseits Ver- 
ben nonadditiver Aspektualität ab. Ist das nicht der Fall, wird Modalität erzeugt. Die werden + Infinitiv-Konstruktionen werden daher vorzugsweise von additiven (imperfektiven) Verben gebildet, da perfektive / nonadditive Verben eine Verweisungskapazität nach vorne - in die Zukunft haben. Daher vermag Nonadditivität bei morphologischen Präsensformen auch ohne andere, auf die Zukunft verweisende Semanteme Zukunftsbezug zu realisieren. Man kann tatsächlich in Abhängigkeit von spezifischen Textsorten genug Belege für nichtmodalisiertes zukünftiges Futur finden. Das formal weniger markierte und daher ökonomischere Präsens pro futuro wird vor allem im gesprochenen Gegenwartsdeutsch favorisiert. Hier muß ich jedoch ergänzend einlenken, denn es hat sich auf Grund meiner Datenauswertungen, die ich der Textsorte Erzählung / Roman und prototypisch für das gesprochene Gegenwartsdeutsch der Textsorte Talkshow entnommen habe, erwiesen, daß morphologische Präsensformen, auch wenn sie mit nonadditiven Verben gebildet werden, nicht durchweg kontextunabhängig zukunftsbezogen sind. Es gibt immer wieder sprachliche und außersprachliche/ suprasegmentale Signale, die den jeweiligen Zeitbezug herstellen oder ihn sogar aufheben können. Es handelt sich um inhaltliche Verweisungen, die sich gerade in textsortenspezifischen Untersuchungen besonders gut ausdifferenzieren lassen. Das, was zum Beispiel im Roman auf der lingualen Ebene geleistet werden muß (wie etwa nichtdritte Personen, entsprechende Zeitadverbien, bestimmte Arten von Nebensätzen oder auch entsprechende lineare Serialisierungen usw. als komplexere grammatische Entitäten, welche den höheren Markiertheitsgrad der merkmallosen synthetischen Präsensformen mit Zukunftsbezug als Ersatzleistung, von der keinesfalls abgesehen werden kann, ausweisen). Anders verhält es sich bei der Textsorte gesprochenes Gegenwartsdeutsch, wo diese erforderliche Markierungssteigerung eben auch extralingual geleistet werden kann. Denken wir dabei nur an den Satz heute tagen in Bruxelles die 12 Außenminister ....

Da das prototypische Präsens das Präsens der additiven, teilbaren und nichtholistischen Verben ist, bezeichnet es einen homogenen Zeitraum, in dem sich sowohl der Standpunkt des Betrachters als auch der Ort des Verbalgeschehens befinden, ist es im Gegensatz dazu klar, meint Leiss, daß sich bei nonadditiven Verben die Aktzeit immer außerhalb des Sprech- und Betrachtzeitraums befindet, daher werden nichtadditive Verben auch mit den Morphemen der Vergangenheitstempora bevorzugt verwendet, denn durch ihre Außenperspektivierung bewirken sie eine Trennung der Lokalisation des Ereignisses / Zustandes von der des Betrachters. Hier ist auch der Grund dafür zu suchen, daß die vergangene temporale Ebene, welche die Merkmale - präsentisch, -futurisch, \pm holistische Semantik und + Außenperspektive verbindet, ganz gut auch mit dem Perfekt realisiert werden kann. Die eben aufgezeigte Dynamik innerhalb des cleutschen Tempussystems verweist auf noch immer andauernde große Turbulenzen und Umkategorisierungsprozesse. Denken wir dabei an die besonders in der Umgangssprache überaus häufige Verwendung des zukunftsbezogenen Präsens oder etwa an die Behauptung von Leiss, wonach das Präteritum von den analytischen Formen des Perfekts zunehmend verdrängt wird, am massivsten wohl im süddeutschen Raum, und mit ihm 
unter gewissen Voraussetzungen - genauer dem haben-Perfekt additiver Verben - synonym ist. Das Präteritum nonadditiver Verben ziehe sich zu Gunsten des Perfekts vor allem in der gesprochenen Sprache zurück.

Bei der Beschreibung des Tempussystems müßte man auf Grund der oben explizierten Erklärungsstrategien die aspektuellen Verhältnisse unbedingt mitberücksichtigen, da sie laut Leiss für das Erzeugen der refferenziellen Ebene der Tempora eine entscheidende Rolle spielen. Das alte Aspektsystem, das noch in den althochdeutschen Sprachdenkmälern durch Aspektpaare, wie wir sie in den slawischen Sprachen vorfinden, gekennzeichnet wurde, ist mittlerweile zerfallen. Bei aspektneutralen Verben können Tempora nämlich übergeneralisierend verwendet werden und sich somit auf den Weg ihrer Grammatikalisierung begeben. Auf diese Weise wird die Herausbildung der neuen analytischen Konstruktionen motiviert. Eine Entwicklungsstrategie, für die Leiss übereinzelsprachliche Geltung beansprucht.

Diese größtenteils auf der Sprachintuition beruhenden Behauptungen von Leiss empirisch zu überprüfen war das Hauptanliegen meiner jüngsten Textanalysen, in denen ich mich diesmal ausschließlich dem grammatischen Parameter Aspekt zuwende. Die Opposition additiv vs. nonadditiv überprüfte ich unter Berücksichtigung der von der NTS explizierten Markiertheitsrelationen und anderer o.a. Postulate, die für syntaktische Erscheinungen Geltung beanspruchen.

Die zentrale Fragestellung läßt sich wie folgt formulieren:

a/ Gibt es eine Korrelation zwischen additiven / markierteren und nonadditiven / weniger markierten Aspektverben und den Distributionstendenzen vom analytischen Futur und futurischem Präsens einerseits sowie dem Präteritum und dem haben- Perfekt andererseits?

b/ Wie zeichnet sich auf dem Hintergrund dieser gemeinsamen Verbalkategorie das Konkurrenzverhalten der merkmalhafteren analytischen und der formal einfacheren synthetischen Tempusformen $a b$ ?

c/ Gibt es diesbezüglich relevante Unterschiede zwischen den Textsorten Erzählung, die durch zwei Romane aus dem 20. Jahrhundert vertreten und stichprobenweise untersucht worden sind (Alfred Döblin, Berlin Alexanderplatz: 12.500 Sätze - untersucht wurden beide Futurvarianten; Ernst Nowak, Addio Kafka: 2.900 Sätze - untersucht wurden beide Varianten zum Ausdruck der Vergangenheit) und die Textsorte Talkshow (RTL EXPLOSIV und TALK IM TURM: zusammen etwa 120 Minuten Sendezeit, produziert wurden ca. 3000 Sätze; erneut sind beide Futurvarianten untersucht worden und CLUB 2 mit etwa 90 Minuten Sendezeit und 2.900 produzierten Sätzen, hier fokusierte sich die Aufmerksamkeit wieder auf beide Varianten zum Ausdruck der Vergangenheit).

d/ Welche Ergebnisse bezüglich der Fortgeschrittenheit der oben besprochenen syntaktischen Prozesse lassen sich aus den Korpusanalysen ableiten? 
Alle in Prozentsätzen ausgewiesenen Werte, die der anliegenden statistischen Datenauswertung zu ențehmen sind, bestätigen die Produktivität der verbalen Aspektualität bei der Erzeugung von temporalen Referenzen im Sinne von Leiss. (Die Unterschiede würden sich sicherlich noch als relevanter erweisen, wenn man bei der Statistik auch die diesbezüglich noch sensibleren Indexe der Verschiedenheit mitberücksichtigte. Diese werden bei der Weiterführung der Untersuchungen eingesetzt, die auch eine Erweiterung der Korpora voraussetzt.)

Es besteht also eindeutig eine Korrelation zwischen additiven und nonadditiven Verben und der Distribution von Tempusformen. Einige Ergebnisse liefern zwar keine Relevanz, jedoch verweist keines auf eine, den Postulaten entgegenwirkende Tendenz. Überraschend sind eigentlich nur die statistischen Ergebnisse, die sich auf die Austauschbarkeit des haben-Perfekts mit dem Präteritum beziehen. Leiss räumt diesbezüglich dem gesprochenen Gegenwartsdeutsch mehr Flexibilität ein. Jedoch sind die beiden Vergangenheitsformen gerade in der Talkshow zu etwa $20 \%$ austauschbar, wogegen im Roman dieser Prozentsatz bei $40 \%$ liegt, was konkret heißt, daß sich gerade in dieser Textsorte das additive Perfekt und das nonadditive Präteritum, die als synonym gelten, prozentuell die Waage halten. Die Begründung hierfür kann man unter anderem auch darin finden, daß in der untersuchten Talkshow die beiden Vergangenheitsformen am Bestand aller Tempusformen nur mit etwa $3 \%$ beteiligt sind, im Roman dagegen mit $25 \%$. Ein solcher Tatbestand verweist auf die Unverträglichkeit der Verwendung vor allem des Präteritums in einer engagierten Besprechung der zumeist auf hier und jetzt bezogenen Ereignisse oder Zustände, wie etwa im besagten Club 2, wo heftig über den Themensschwerpunkt diskutiert wird, ob Krankenschwester zu sein, ein Traumberuf oder ein Alptraumberuf sei. Das Perfekt ist hier wesentlich üblicher als das Präteritum, wie man das den Handouts entnehmen kann, weil es zu über $60 \%$ häufiger auftritt als im Roman.

Für das gesprochene Gegenwartsdeutsch ist daher anzunehmen, daß es erwartungsgemäß prototypischer ist für das haben-Perfekt, daher finden hier auch die untypischeren additiven Verben massiveren Zugang zu dieser Konstruktion, als das beim Präteritum für seine untypischere Variante - die nonadditiven Verben - der Fall ist. Das Präteritum erwies sich in der Textsorte Erzählung als die nach wie vor üblichere Oberflächenrealiserung der Zeitsphäre vergangen. Das Gleiche gilt auch für die nichtmodalen werden + Infinitiv Konstruktionen als dem grammatikalisierten Futur im Deutschen.

Der Umstand, daß auch nonadditive Verben massiven Zugang sowohl zum Perfekt als auch zum analytischen Futur haben, bestätigt einerseits die These, daß beide analytischen Konstruktionen schon als Aktualisierungsvarianten sowohl der imperfektiven als auch der perfektiven Vergangenheit (Perfekt) und der nichtmodalisierten Zukunft grammatikalisiert worden sind. Andererseits aber auch die Verdrängungsthese des Präteritums durch das Perfekt, denn sie sind auch in der weniger veränderungsfreundlichen Textsorte Erzählung zu fast $40 \%$ weitgehend synonyme Varianten. Dieser 
Proze $\beta \mathrm{mu} ß$ auch schon relativ weit fortgeschritten sein, denn die in beiden Textsorten vorgefundenen Unterschiede sind nicht derart gravierend, um die Annahme zu rechtfertigen, daß der besagte Prozeß erst allmählich einsetzt. Auch was die Markiertheitsverhältnisse anbelangt, kann festgestellt werden, daß beim analytischen Perfekt, welches hauptsächlich verschiedene Präsensvarianten zum temporalen Hintergrund hat, dieses temporale "shifting" durch die formale Merkmalhaftigkeit gekennzeichnet wird, weitere Markierungen sind redundant, daher ist die Konstruktion auch im gesprochenen Gegenwartsdeutsch ökonomisch genug, und wird entsprechend häufig verwendet. Beim zukunftsbezogenen Präsens hingegen wird die erforderliche Ersatzmarkierung durch die auf den ersten Blick weniger transparenten außersprachlich angelegten situativen Impulse geleistet. Diese kann man besonders effizient in natürlichen Gesprächsabläufen realisieren, wo sie auch überzeugend häufiger vorzufinden sind als ihre umständlichere analytische Variante.

Wiederum kann man also feststellen, daß sich sprachliche Vorgänge stark von Ökonomieprinzipien motivieren lassen.

\section{STATISTISCHE DATENAUSWERTUNG:}

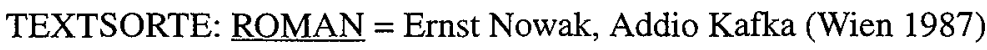

$\begin{array}{llll} & & 1 & 2 \\ \text { haben-Perfekt : } & 12,5 \% & \mathrm{~A}=39,3 \% & / \mathrm{N}=60,7 \% \\ \text { Präteritum : } & 87,5 \% & \mathrm{~A}=63,2 \% & / \mathrm{N}=36,8 \%\end{array}$

- mit $25 \%$ am Bestand aller Tempusformen im Korpus vertreten

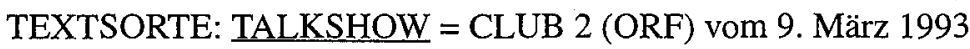

haben-Perfekt : $\quad 80 \% \quad \mathrm{~A}=42,1 \% \quad / \mathrm{N}=57,9 \%$

Präteritum: $\quad 20 \% \quad \mathrm{~A}=84,2 \% \quad / \mathrm{N}=15,8 \%$

- mit $3 \%$ am Bestand aller Tempusformen im Korpus vertreten

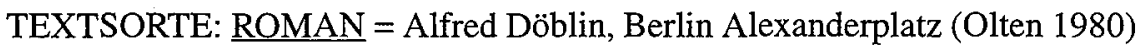

werden + Inf: $\quad 28 \% \quad \mathrm{~A}=45,1 \% \quad / \mathrm{N}=54,9 \%$

Präsens pro futuro: $\quad 72 \% \quad \mathrm{~A}=27,6 \% \quad / \mathrm{N}=72,4 \%$

- mit $6 \%$ am Bestand aller Tempusformen im Korpus beteiligt

TEXTSORTE: TALKSHOW = RTL-Explosiv vom 25. Februar 1993, Talk im Turm SAT 1 vom 31. Jan. 1993

$\begin{array}{llll}\text { werden + Inf:: } & 11,4 \% & \mathrm{~A}=68 \% & / \mathrm{N}=32 \% \\ \text { Präsens pro futuro: } & 88,6 \% & \mathrm{~A}=43,3 \% & / \mathrm{N}=56,7 \%\end{array}$

- mit $8 \%$ am Bestand aller Tempusformen im Korpus vertreten

$1=$ additive Verben

$2=$ nonadditive Verben 


\section{ZUSAMMENFASSUNG}

Die von Leiss (1992:226) aufgestellte These über die Dreigliedrigkeit des Tempussystems im Deutschen wird mit den vom slowenischen Modell der Natürlichen Syntax aufgestellten Postulaten konfrontiert, wonach sich der syntaktische Wandel im Durchsetzen entweder verstärkter oder geschwächter Konstruktionen durchsetzt. Beide Entfaltungsmöglichkeiten sind in spezifischer Weise regelgeleitet.

Der Themenschwerpunkt ist auf zwischenkategorialen Affinitäten fokussiert sowie der textsortenspezifischen Bedeutung des Ko- und Kontextes und anderer diskursiver Elemente zur Herstellung von semantischen Rollen.

Die aufgestellten Postulate werden am sprachlichen Material ausgewertet, welches die Textsorten deutsche Sprechsprache der Gegenwart (TALKSHOWS) und deutschsprachiges literarisches Schaffen (Romane) durch die Kategorie des Tempus (analytisches Futur - Präsens mit Zukunftsbezug; Präteritum - h a b e n Perfekt) miteinander konfrontiert.

\section{LITERATUR}

Comrie, Bernard: Aspect. Cambridge 1976.

Döblin, Alfred: Berlin Alexanderplatz. Olten 1980.

Dressler, Wolfgang U.: Grundfragen der Morphonologie, Wien 1970

Jakobson, Roman: Kindersprache, Aphasie und allgemeine Lautgesetze. Frankfurt/M. 1969.

Jakobson, Roman: Selected Writings II. The Hagü, Paris 1971.

Leiss, Elisabeth: Die Verbalkategorien des Deutschen. Berlin 1992.

Mayerthaler, Willi: Morphologische Natürlichkeit. Wiesbaden 1991.

Nowak, Ernst: Addio, Kafka. Wien 1987.

Orešnik, Janez: Periphrasen sind verstärkte Konstruktionen. In: Beiträge zum 5. Essener Kolloquium über "Grammatikalisierung, Natürlichkeit und Systemökonomie", Bochum 1990.

Teržan-Kopecky, Karmen: Kategorialer Entfaltungsprozess im Lichte seiner distinktiven Merkmale. In: Untersuchungen zum Deutschen als Fremd- und Zweitsprache. Graz 1992.

Wurzel, Wolfgang U.: Flexionsmorphologie und Natürlichkeit. In: Studia grammatica XXI, Berlin 1984. 
V članku se soočajo teza E. Leiss (1992:226) o tročlenskosti časovnega sistema v nemščini in postulati slovenskega modela naravnostne skladnje, po katerem se uveljavlja skladenjsko spreminjanje bodisi okrepljenih bodisi ošibljenih konstrukcij. Obe možnosti razvoja potekata po pravilih na specifičen način.

Tematsko težišče je osredotočeno na medkategorialne afinitete kot tudi na pomen ko- in konteksta, specifičnega za besedilno vrsto, in drugih diskurzivnih elementov za oblikovanje semantičnih vlog. Vrednotenje postulatov je potekalo na jezikovnem materialu, ki med sabo sooča besedilne vrste moderni nemški govorni jezik (TALKSHOWS) in nemškogovoreče literarno ustvarjanje (romani) preko kategorije časa (analitični futur - prezent z ozirom na prihodnost, preterit - perfekt "haben"). 